\title{
Astragaloside IV attenuated TGF- $\beta 1$ - induced epithelial-mesenchymal transition of renal tubular epithelial cells via connexin43 and Akt/mTOR signaling pathway
}

\section{Yonghong Lian}

GuangXi University of Chinese Medicine https://orcid.org/0000-0002-6699-332X

\section{Cuiqiong Li}

First People's Hospital of Liangshan Yi Autonomous Prefecture

Jianchun Li

Affliated Traditional Medicine Hosipital of Southwest Medical University

\section{Yongxiang Xie}

Guangxi Traditional Chinese Medical University Affiliated First Hospital

Qiancheng Liu

Guangxi Traditional Chinese Medical University Affiliated First Hospital

\section{Minhua Wu}

Guangxi Traditional Chinese Medical University Affiliated First Hospital

\section{Wei Shi}

Guangxi Traditional Chinese Medical University Affiliated First Hospital

\section{Lifeng Meng ( $\nabla$ mlf428@126.com )}

First Affiliated Hospital of Guangxi University of Chinese Medicine

\section{Research}

Keywords: Astragaloside IV, connexin43, Akt/mTOR signaling pathway, epithelial-mesenchymal transition, renal tubular epithelial cells

Posted Date: November 2nd, 2021

DOI: https://doi.org/10.21203/rs.3.rs-1026692/v1

License: (c) (i) This work is licensed under a Creative Commons Attribution 4.0 International License. Read Full License 


\section{Abstract}

INTRODUCTION: The objective of the study was to observe whether Cx43 could regulate EMT of RTECs by influencing Akt/mTOR signaling pathway, and whether ASV could inhibit the development of renal interstitial fibrosis by regulating $\mathrm{Cx} 43$.

METHODS: Lentivirus infection was transfected into RTECs with the final concentration of $50 \times P F U /$ cell to regulate the expression of Cx43.And RTECs were intervened by different doses of ASV. After synchronous culture of RTECs in each group,cell morphological changes were observed and the expression levels of EMT-related indicators, and the expression levels of $\mathrm{Cx} 43$, the protein expressions and phosphorylation levels AKT and mTOR in different groups were detected by WB.

RESULTS: When the expression of Cx43 in RTECs was regulated by lentivirus infection, the degree of EMT induced by TGF- $\beta 1$ and the phosphorylation level of Akt and mTOR were changed accordingly, indicating that Akt/mTOR pathway might be a downstream molecular mechanism by which $\mathrm{C} \times 43$ could regulate EMT. After intervention with different doses of ASV, the expression level of Cx43 increased with obvious concentration dependence, and the expression levels of p-Akt and p-mTOR were significantly altered, suggesting that ASV could effectively increase the protein expressions of TGF- $\beta 1$-induced Cx43 in RTECs and inhibit the phosphorylation levels of Akt and mTOR.

CONCLUSION: Cx43 is the main material basis of RTECs'injury, and ASV could inhibit TGF- $\beta 1$ induced RTECs transdifferentiation. In-depth study of the mechanism may provide a broad application prospect for the treatment of renal interstitial fibrosis.

\section{Introduction}

End-stage renal disease (ESRD) is the final stage of various chronic kidney diseases, which refers to the irreversible decline of renal physiological functions. ESRD is characterized by high disability rate, high mortality rate and high medical costs. ESRD makes patients only rely on renal replacement therapy (kidney transplantation and dialysis therapy) to maintain the pathological state needed for normal life [1]. The incidence of renal failure caused by chronic kidney disease and other chronic diseases is increasing year by year worldwide, leading to the continuous increase in the number of patients with ESRD, which has become an increasingly serious public health problem. By the end of 2012, more than 3 million patients worldwide were receiving treatment for ESRD, and the rate is increasing at $7 \%$ per year, far exceeding the world population growth rate [2]. In recent years, the incidence of ESRD in China has been increasing year by year and the growth rate has accelerated, causing economic losses and depletion of health resources to the country, but also bringing heavy economic burden to the whole society, patients and their families [3].

Renal interstitial fibrosis (RIF) is almost the common manifestation and pathological basis of all kinds of chronic renal disease in the late stage. The occurrence of RIF is mainly manifested by the production of a large number of fibrogenic factors and the proliferation of fibroblasts. Epithelial mesenchymal 
transformation (EMT) is one of the main causes of cell proliferation and subsequent renal fibrosis [4].EMT is a transdifferentiation process in which interepithelial cells disadhere, polarity disappears, and expression of epithelial marker proteins is reduced to obtain mesenchymal cell phenotypes under specific physiological and pathological conditions, such as embryonic development, histopathogenesis, and the pathogenesis of a variety of chronic diseases. Transforming growth factor (TGF- $\beta 1$ ) is a fibrogenic growth factor and a key mediator in the induction of progressive renal failure due to multiple renal injuries [5].TGF- $\beta 1$, a member of the TGF family, is a multifunctional peptide cytokine associated with a variety of biological processes. It can regulate the genesis and differentiation of tissue morphology through such pathways as cell proliferation, differentiation, metastasis, adhesion, apoptosis and the synthesis and secretion of extracellular matrix, including the regulation of EMT in many different epithelial cells.

Multiple signaling pathways are involved in TGF- $\beta 1$-induced renal tubular epithelial EMT. TGF- $\beta 1$ induced EMT in renal tubular epithelial cells can be mediated through the classical TGF- $\beta$ /Smads pathway, and non-Smad-dependent downstream TGF- $\beta 1$ pathways also play an important role in the occurrence and development of EMT, among which the PI3K/ Akt /mTOR signaling pathway plays an important role in cell metabolism, apoptosis, proliferation and differentiation [6].Gap junctions constitute inter-cell communication, which can mediate the transfer of small molecular signals between adjacent cells, participate in the material and information exchange between cells, and then regulate a variety of physiological processes of cells [7].Cx43 is one of the most widely expressed members of the Connexin (Cx) family, and is also a molecule that has been studied extensively at present. Studies have found that the expression and functional changes of $\mathrm{Cx} 43$ are closely related to renal fibrosis [8].Studies have shown that the activity of TGF- $\beta$ is mediated by CX43, which can increase the phosphorylation level of Smad2, thereby regulating the TGF- $\beta /$ Smads pathway [9].However, this study by Stains JP et al. [10] found that in osteoblasts, changes in CX43 content could cause changes in PI3K/ Akt /mTOR pathway activity. Therefore, whether CX43 could also mediate TGF- $\beta$ activity through PI3K/ Akt /mTOR pathway remains to be further studied.

Astragaloside IV (ASV) is the main effective component of Astragalus membranaceus, which has various pharmacological effects such as immunomodulatory, antibacterial, antiviral, antioxidant, scavenging oxygen free radicals, protecting cell membrane and so on [11].Studies have shown that ASV can alleviate renal tubulointerstitial fibrosis and is closely related to the inhibition of TGF- $\beta$ /Smad signaling pathway [12].Other studies have found that ASV can inhibit gastric cancer cell transdifferentiation induced by $\mathrm{PI}$ IK/ Akt /NF- kB pathway which is activated by TGF- $\beta 1$ [13]. Whether ASV could mediate the activity of $\mathrm{PI} 3 \mathrm{~K} / \mathrm{Akt} / \mathrm{mTOR}$ by regulating the expression of connexin 43 , and then affect the transdifferentiation of renal tubular epithelial cells has not been studied,

In this study, TGF- $\beta$ 1-induced transdifferentiation of renal tubular epithelial cells was used as the research object. Low, medium and high concentrations of ASV were intervened to detect the protein and mRNA expression levels of E-cadherin, a-SMA and vimentin, and further to detect the protein and mRNA expression levels of connexin 43 and the activity of PI3K/Akt/mTOR pathway. The research aim is to 
investigate the effect of ASV on TGF- $\beta 1$-induced renal tubular epithelial cell transdifferentiation and its possible mechanism.

\section{Materials And Methods \\ 2.1 Materials}

Rat tubular epithelial NRK-52E cell line were obtained from the Cell Bank of the Chinese Academy of Sciences (Shanghai, China); Transformational growth factor $\beta 1$ (TGF - $\beta 1$ ) was purchased from R\&D Systems, Inc. (Minneapolis, MN); Fetal bovine serum (FBS) were provided by Invitrogen (Grand Island, NY); RPMI nad Dulbecc's modified eagle's medium (DMEM) were supplied by GIBCO (gaithersburg,MD); Dimethyl sulfoxide (DMSO) and TRIzol reagent, Anti-Cx43 antibody, RIPA, phenylmethlysulfonyl fluoride, Anti E- cadherin and Anti beta actin, and Anti Alpha- SMA were puschased from Sigma chemical Co. (St. Louis, MO), Anti-Akt 1/2/3 (H-136), Anti-phospho-Akt 1/2/3 (ser 473) Anti-PTEN antibodies, anti- FOXO antibodies, antibodies against phospho PI3K-p110a were bought from Santa Cruz Biotechnology (Santa Cruz, CA), Anti-phospho-m-TOR was from Cell Signalling (Beverly, CA).

\subsection{EMT model of RTECs induced by TGF- $\beta 1$}

RTECs were cultured with conventional methods. When RTECs grew to fusion of $80 \%-90 \%$, digestion was performed with trypsin and DMEM/F12 medium containing 100\% FBS was added to terminate digestion. Counting with a counter plate, the cells were adjusted to an appropriate concentration, and inoculated into six-well culture plates. When the cell confluence reached $60 \%$, drug stimulation was added, and the cells were kept hungry 24 hours before treatment. According to relevant literature and preliminary experiments, $6 \mathrm{ng} / \mathrm{m} 1$ TGF- $\beta 1$ was used to stimulate renal tubular epithelial cells for 48 hours so as to construct the EMT model of RTECs[14]. Cellular immunofluorescence was used to observe the changes in cell morphology, and Western-blot were used to detect the protein levels of EMT-related indicators to identify the EMT model.

\subsection{Construction and packaging of lentivirus vectors}

With reference to GenBank, GJA1 gene interference sequence was designed and synthesized by Shanghai Genechem Co., LTD. Then, recombinant lentivirus clones with overexpression and interference were prepared with GJA1 gene and control lentivirus as targets. Simply, overexpressed lentivirus transfer vectors (GV208) and lentivirus transfer vectors (GV112) were constructed. Both vectors contain ampicillin resistance gene and enhanced green fluorescent protein gene. For overexpressed lentivirus vectors, homologous recombination sequence should be added to the 5 'end of the amplification primers. For interfering lentivirus vectors, the primers were designed to add restriction sites at both ends. The packaging of the virus was accomplished by transient transfection with 293T cells carrying the transfer plasmid and three packaging vectors: the tool carrier plasmid carrying the target gene or target sequence, and pHelper 1.0 and pHelper 2.0. Lentivirus particles were collected 3 days after transfection and concentrated at $25000 \mathrm{rpm}$ for 2 hours at $4^{\circ} \mathrm{C}$. With discarding the supernatant, the virus preservation 
solution was added and fully dissolved. Centrifuged at a high speed of 10000 RPM, the supernatant was taken and packed according to the requirements, and then the lentivirus quality was detected.

\subsection{Transfecting into renal tubular epithelial cells with lentivirus}

The RTECs in the logarithmic growth stage was digested with trypsin, and the complete medium was added to make $3-5 \times 104 / \mathrm{mL}$ cell suspension, and lentivirus infection was transfected with the final concentration of 50xPFU/ cell. The cells were divided into 5 groups: (1) non-infected HRTECs $+6 \mathrm{ng} / \mathrm{m} 1$ TGF- $\beta 1$ (TGF- $\beta 1$ group); (2)RTECs infected with lentivirus shRNA-Con +6ng/m1 TGF- $\beta 1$ (shRNA-Con group); (3)HRTECs infected with lentivirus shRNA-Cx43+6ng/m1 TGF- $\beta 1$ (shRNA-Cx43 group); (4)RTECs infected with lentivirus OE-con $+6 \mathrm{ng} / \mathrm{m} 1$ TGF- $\beta 1$ (OE-Con group); (5)RTECs infected with lentivirus OE$\mathrm{Cx} 43+6 \mathrm{ng} / \mathrm{m} 1 \mathrm{TGF}-\beta 1$ (OE-Cx43 group). After infection, the cells were cultured in conventional medium for about 8-16h. GFP expression was observed under a fluorescence microscope, and when the infection efficiency reached about $80 \%$,the cells were collected and protein and gene levels of EMT-related indicators were detected.

\subsection{Evaluating the effective concentration of ASV by CCK-8 method}

Cell count kit -8 (CCK-8) was performed to assess the toxicity of ASV. Briefly, $5 \times 10^{3}$ RTECSs in logarithmic phase were distributed in 96 -well plates and kept in an incubator at $37^{\circ} \mathrm{C}$ under $5 \% \mathrm{CO}_{2}$ for 24 hours. After cells treated with various concentrations of ASV (ranging from 0 to $120 \mu \mathrm{g} / \mathrm{ml}$ ) for 48 hours, $10 \mu \mathrm{L}$ of CCK-8 solution (Beyotime, China) was added to each well and the plates were incubated for 3 hours. Microplate reader (Bio-Tek, USA) was used to record the absorbance at $450 \mathrm{~nm}$ and to detect the cell activities in RTECs under different concentrations of ASV.

\subsection{ASV intervention program in vitro and index detection:}

In order to study the effect of ASV on TGF- $\beta 1$-induced EMT, RTECs were divided into 5 groups according to different intervention methods: the normal group ( $10 \%$ fetal bovine serum), the model group $(6 \mathrm{ng} / \mathrm{ml}$ TGF- $\beta 1$ ), low-dose of ASV group ( $6 \mathrm{ng} / \mathrm{ml} \mathrm{TGF-} \beta 1+10 \mu \mathrm{g} / \mathrm{ml}$ ASV), medium-dose of ASV group ( $6 \mathrm{ng} / \mathrm{ml}$ TGF- $\beta 1+40 \mu \mathrm{g} / \mathrm{ml}$ ASV) and high-dose of ASV group ( $6 \mathrm{ng} / \mathrm{ml} \mathrm{TGF-} \beta 1+80 \mu \mathrm{g} / \mathrm{ml}$ ASV). After synchronous culture of RTECs in each group at $37^{\circ} \mathrm{C}$ in $5 \% \mathrm{CO}_{2}$ incubator for 24 hours, cell morphological changes were observed and the expression levels of EMT-related indicators, such as Ecadherin and $\alpha$-SMA were detected, and the expression levels of $\mathrm{Cx} 43$, the protein expressions and phosphorylation levels AKT and mTOR in different groups were detected by WB.

\subsection{Statistical analysis}

The experimental data were expressed as mean \pm standard deviation (SD), and SPSS20.0 statistical software was used for statistical processing. The measurement data were analyzed by one-way ANOVA, 
and independent sample t test was used for multiple comparisons of the two sample means. Statistical significance was indicated at a level of $\mathrm{P}<0.05$.

\section{Results}

\subsection{Establishment of EMT model of RTECs induced by TGF- $\beta 1$}

RTECs were observed under fluorescence microscope, and the results were shown in Figure 1. As a marker for epithelial cells, the expression of E-cadherin in the normal control group was abundant and continuously distributed inside and around the cells, while the expression of a-SMA, as a marker for stromal cells, was almost absent. After $6 \mathrm{ng} / \mathrm{ml}$ TGF- $\beta 1$ stimulated cells for $48 \mathrm{~h}$, E-cadherin in the model group was fractured and the expression level significantly decreased compared with the normal group ( $P$ $<0.01$ ) (Figure 1-a, b), and the expression level of a-SMA significantly increased $(P<0.01)$ (Figure 1-a, b). All these indicated that RTECs induced by TGF- $\beta 1$ should lose the epithelial cell morphology and transform into a characteristic mesenchymal phenotype.

\subsection{Lentiviral vector transfection of RTECs in vitro effectively regulated $\mathrm{Cx} 43$ expression}

Lentiviral vector was transfected into RTECs to interfere with Cx43 specifically and the effect was observed under immunofluorescence microscope. The expression of Cx43 in shRNA-Cx43 group transfected with lentiviral vector was significantly decreased compared with the model group $(P<0.001)$, while shRNA-Con group, which was the negative control group, showed no significant change in $\mathrm{Cx} 43$ expression compared with the model group ( $>>0.05$ ) (Figure 2-a, b). In the OE-Cx43 group transfected with overexpressed lentivirus-interfering vector, the expression of $\mathrm{Cx} 43$ was significantly increased compared with the model group $(\mathrm{P}<0.001)$, while in the $\mathrm{OE}-\mathrm{Cx} 43$ group, the expression of $\mathrm{Cx} 43$ was not significantly changed $(P>0.05)$ (Figure 2-a, b). Western blotting also confirmed the process, which indicating that the lentivirus interference could effectively regulate the expression of $\mathrm{Cx} 43$ (Figure 2-C, $d, e, f)$.

\subsection{Up-regulation of Cx43 could inhibit TGF- $\beta 1$-induced EMT in RTECs}

The lentivirus shRNA-Cx43 was used to decrease the expression of $\mathrm{Cx} 43$. Immunofluorescence results showed that compared with the model group, E-cadherin expression was significantly decreased in shRNA-Cx43 group $(P<0.05)$ (Figure $3-a, b)$, while a-SMA expression was significantly increased in shRNA$\mathrm{Cx} 43$ group $(\mathrm{P}<0.05)$ (Figure $3-\mathrm{a}, \mathrm{C})$. These were consistent with the changes in the expression of each indicator detected by western blot (Figure 3- d,e,f), indicating that when the expression of Cx43 was decreased, the degree of EMT induced by TGF- $\beta 1$ in RTECs was more obvious. On the contrary, lentivirus 
OE-Cx43 was used to reduce the expression of $\mathrm{Cx} 43$. Compared with the model group, the expression of $\mathrm{E}-$-cadherin in OE-Cx43 group increased significantly $(\mathrm{P}<0.05)$ (Figure $3-\mathrm{a}, \mathrm{b})$, and the expression of $a-S M A$ decreased significantly $(P<0.05)(F i g u r e 3-a, c)$. These were consistent with the changes in the expression of each indicator detected by western blot (Figure 3- d,e,f), indicating that when $\mathrm{Cx} 43$ expression was upregulated, the degree of EMT induced by TGF- $\beta 1$ was inhibited. All these suggested that $C \times 43$ might be an important regulatory molecule involved in the process of EMT induced by TGF- $\beta 1$.

\subsection{Akt/mTOR pathway might be a downstream molecular mechanism by which $\mathrm{Cx} 43$ regulates the occurrence of EMT.}

Under the immunofluorescence microscope, it was found that when the expression of Cx43 was reduced by lentiviral vector transfected into RTECs, the phosphorylation level of Akt and mTOR were significantly increased compared with the model group (Figure 4-a,b,c), and the differences were statistically significant $(P<0.05)$. These were consistent with the changes in the expression of each indicator detected by western blot (Figure 3-d,e,f), which suggested that the EMT induced by TGF- $\beta 1$ in RTECs mignt be related to the activation of Akt/mTOR pathway when the expression of $\mathrm{Cx} 43$ was decreased. However, when the expression of $\mathrm{Cx} 43$ was increased by lentiviral vector transfection, the phosphorylation level of Akt and mTOR were significantly reduced in the OE-Cx43 group compared with the model group (Figure $3-a, b, c)$, with statistically significant differences $(P<0.05)$. These were consistent with the changes in the expression of each indicator detected by western blot (Figure 3-d,e,f) ,which suggested that the EMT induced by TGF- $\beta 1$ in RTECs was alleviated when Cx43 expression was up-regulated, which might be related to the inhibition of Akt/mTOR pathway.

\subsection{Determination of the effective concentration of ASV}

CCK-8 assay was employed to examine the effects of ASV with different concentration $(10,20,40,80$, $120 \mu \mathrm{g} / \mathrm{ml}$ ) on RTECs' cell viability. The cell viability of the normal control group was defined as $100 \%$, and the ratio to the normal control group was the cell viability of the group stimulated by ASV at different concentrations. When the concentrations of ASV were from 10 to $80 \mu \mathrm{g} / \mathrm{ml}$, the cell activities increased or decreased compared with the normal control group, but the differences were not statistically significant $(P>0.05)$. When the concentration of ASV was $120 \mathrm{~g} / \mathrm{ml}$, the cell viability was significantly reduced and the difference was statistically significant $(P<0.05)$ (Figure 5). Therefore, $10 \mu \mathrm{g} / \mathrm{ml}, 40 \mu \mathrm{g} / \mathrm{ml}$ and $80 \mu \mathrm{g} / \mathrm{ml}$ were selected as the low, medium and high intervention concentrations for ASV within the concentration range.

\subsection{ASV improved EMT induced by TGF- $\beta 1$ in RTECs}

To investigate whether ASV plays a role in reducing TGF- $\beta 1$ induced EMT in RTECs, immunofluorescence assay was used to detect the expression of EMT- related marker proteins E-cadherin and a-SMA. Compared with the model group, the expression levels of $\mathrm{Cx} 43$ and $\mathrm{E}$-cadherin increased after intervention with $10 \mu \mathrm{g} / \mathrm{ml}$ of ASV (figure 6-a,b,c), while the expression levels of a-SMA decreased (figure 
6-a,d), but the difference was not statistically significant $(P>0.05)$. After intervention with $40 \mu \mathrm{g} / \mathrm{ml}$ and $80 \mu \mathrm{g} / \mathrm{ml}$ of ASV, compared with the model group, the expression levels of Cx43 and E-cadherin increased (Figure 6-a,b,c) $(P<0.05)$, and the expression levels of a-SMA decreased (Figure 6-a,d) $(P<0.01)$, with statistically significant differences. Consistent with the immunofluorescence results, the effect was also confirmed by western blotting (Figure 6-e,f,g). The above experiments indicated that ASV culd effectively inhibit TGF- $\beta 1$ - induced EMT in RTECs.

\subsection{ASV reversed the expression of Cx43 and the disorders in Akt/mTOR signaling pathway.}

To further clarify whether ASV affects the transdifferentiation of RTECs cells via interfering with the Cx43 and Akt/mTOR pathways, western blotting was used to detect the expression of $\mathrm{Cx} 43$, the levels of Akt and mTOR proteins and their phosphorylation. The protein expressions of $\mathrm{Cx} 43$ was shown in Figure 6-i and Figure 6-I. Compared with the normal control group, the expression level of $\mathrm{Cx} 43$ in the model group was significantly decreased after $6 \mathrm{ng} / \mathrm{ml}$ TGF- $\beta 1$ stimulated for $48 \mathrm{~h}$. While, after intervention with lowdose, medium dose and high-dose of ASV, the expression levels of Cx43 increased compared with the model group, and the effect showed obvious concentration dependence. As shown in Figure <link rid="fig6" $>6</$ link $>-i$, $<$ link rid="fig6" $>6</$ link $>-j$ and $<$ link rid="fig6" $>6</$ link $>-k$, there were no significant differences in the protein expressions of Akt and mTOR in the normal group, model group, low-dose of ASV group, medium-dose of ASV group, and high-dose of ASV group, while the protein expressions of $p$ Akt and p-mTOR were significantly changed. Compared with the normal control group, p-Akt /Akt and p$\mathrm{mTOR} / \mathrm{mTOR}$ were increased in the model group, while the ratios of $\mathrm{p}$-Akt/Akt and p-mTOR/mTOR were slightly decreased after intervention with low-dose and medium-dose of ASV, with no statistically significant difference $(P>0.05)$. While after intervention with high-dose of ASV, $p$-Akt/Akt and p-mTOR /mTOR were significantly reduced, and the difference was statistically significant $(p<0.01)$. The above experiments indicated that ASV could effectively increase the protein level of TGF- $\beta 1$-induced Cx43 and inhibit the phosphorylation level of Akt and its downstream molecule mTOR.

\section{Discussion}

According to modern medicine, RIF is characterized by degeneration and loss of renal tubule and accumulation of extracellular matrix, which is involved with TGF- $\beta 1$, angiotensin II, HER2 and other biological factors. As a fibrogenic factor, TGF- $\beta 1$ can induce the activation of myofibroblasts and inhibit the degradation of collagen fibers, which resulting in the accumulation of large amounts of collagen fibers between tissues[15]. Studies have shown that TGF- $\beta 1$ plays an initial activation role in the formation of fibrosis. TGF- $\beta 1$ can bind to receptors on the cell surface, activate multiple cell signaling pathways, and induce the expression of target genes, thus promoting the generation of fibrosis [16].

TGF- $\beta 1$ has been shown to rapidly activate PI3 kinase in a variety of cellular systems, leading to activation of Akt kinase [17].Akt is a central regulator of multiple pathways involved in cell survival, cell size control, and cell migration. Akt regulates cell functions by phosphorylating a variety of downstream 
factors such as enzymes, kinases and transcription factors. Inhibitors of PI3 kinase and Akt, or a dominant negative form of Akt, have been found to inhibit TGF- $\beta 1$-induced EMT[18]. mTOR is a serine/threonine protein kinase that regulates cell growth, cell proliferation, cell movement, protein synthesis and transcription. Over-activated mTOR promotes EMT, and MTOR inhibitors inhibit bleomycininduced pulmonary fibrosis and EMT in mice [19].

The EMT changes of RTECs are closely related to the damage of gap junction intercellular communication(GIC). GJIC mediated by $\mathrm{Cx} 43$ is involved in the transmission of energy and information between cells, and plays an important role in the regulation of cell proliferation, differentiation, metabolism and internal environment stability and other physiological processes [20]. Impaired intercellular information transmission can lead to decreased cell proliferation, cell cycle arrest and cell hypertrophy, and then increase secretion of extracellular matrix, which eventually leads to the occurrence of renal interstitial. The phosphorylation of CX43 directly affects the assembly, degradation of CX43 on the cell membrane and the GJIC mediated by gap junctions. There are at least five serine phosphorylation sites in connexin 43 , which are necessary for the aggregation and intercellular junction of connexin 43 . It has been proved that there are action sites of protein kinase $C$ and mitogen activated protein kinase on the molecule of connexin 43 , which are related to the closure of gap junction channels [21].

James $\mathrm{CC}$ et al. [22] proved that during the process of cell transdifferentiation, the reduction of $\mathrm{Cx} 43$ protein can act as a key link to activate PI3K/ Akt /mTOR to further promote the occurrence of cell transdifferentiation. Studies have shown that the glomerular mesangial cells exposed to high concentration of glucose could reduce CX43, inhibit PTEN, trigger Akt phosphorylation, activate downstream mTOR, and lead to GMC hypertrophy [23]. On the contrary, overexpression of CX43 could inactivate PTEN/PI3K/Akt/M-TOR pathway, prevent Akt and mTOR phosphorylation, and reverse GMC hypertrophy [23]. Kuang Jy et al. [24] believed that the negative correlation between CX43 expression and Akt/ ERK activation was caused by the direct interaction between Akt/ ERK and CX43. They found that the T286/A305/Q308/Y313 residues and S272/S273 at the carboxyl terminus of CX43 were crucial for its binding to Akt and ERK, respectively. In this study, Western Blot, immunofluorescence and other studies confirmed that up-regulation of CX43 could inhibit the TGF- $\beta 1$-induced EMT process in RTECs. We further observed that when lentivirus shRNA-CX43 was used to decrease the expression of CX43, the phosphorylation levels and gene expressions of Akt and mTOR were significantly increased, whereas when lentivirus OE-CX43 was used to increase the expression, the phosphorylation levels and gene expressions of Akt and mTOR were significantly decreased. All these indicate that Akt/mTOR signaling pathway may be the downstream molecular mechanism of CX43 regulating EMT. ASV has specific inhibitory effects on cell transdifferentiation and tissue fibrosis, and its mechanism involves complex signaling pathways.

Studies have shown that ASV inhibits PI3K/Akt/mTOR signal and prevents epithelial mesenchymal transdifferentiation (EMT) process by down-regulating TGF- $\beta 1$ expression [25].In this study, different concentrations of ASV were used to intervene TGF- $\beta 1$-induced Rtecs cell transdifferentiation. Compared with the model group, ASV could reduce the expression of a-SMA, vimentin and other genes and proteins, 
and enhance the expression of E-cadherin molecule, indicating that ASV had anti-transdifferentiation effect, which was consistent with the existing studies. After drug intervention, the expression level of CX43 was increased and the phosphorylation level of PI3K/ Akt /mTOR was decreased in a dosedependent manner. Combined with existing studies, it was speculated that the inhibition of TGF- $\beta 1$ induced RTECs cell transdifferentiation by ASV might be related to the inhibition of phosphorylation of Akt and mTOR by regulating the expression of CX43 molecule.

\section{Conclusion}

In conclusion, the signaling pathways involved in cell transdifferentiation are complex and diverse. In this study, we focused on the relations between expression of CX43 and the activation status of $\mathrm{PI3K} / \mathrm{Akt} / \mathrm{mTOR}$ pathway. Gap junction proteins (GJPs) play an important role in the pathological processes of cells, tissues and organs. The gap junction protein family, especially the CX43 molecule, has been closely associated with the development of disease. CX43 is the main material basis of functional impairment of RTECs. Although it has been found that CX43 molecule is the upstream regulator of $\mathrm{PI} 3 \mathrm{~K} / \mathrm{Akt} / \mathrm{mTOR}$ signaling pathway, relevant studies still need to be further improved in this study. Further study on the mechanism of its action on intercellular junction communication may provide a new idea for the development of drugs to prevent EMT of renal tubular epithelial cells by regulating CX43.

In this study, it was found that ASV could indeed affect the expression of molecules on the $\mathrm{PI} 3 \mathrm{~K} / \mathrm{Akt} / \mathrm{mTOR}$ signaling pathway by regulating CX43, but this may only be a part of the mechanism of ASV inhibiting TGF- $\beta 1$-induced RTECs'transdifferentiation, which can only be regarded as a certain theoretical support. As for whether it can be used in the clinical treatment of renal interstitial fibrosis, it still needs to be further improved in vivo experiments and clinical studies. ASV has a variety of pharmacological activities, in-depth study of its repair mechanism of renal tubular epithelial cell damage, as well as the improvement of clinical trials, may provide a broad application prospect for the treatment of ESRD against renal interstitial fibrosis.

\section{Declarations}

\section{Acknowledgements}

Not applicable

\section{Authors'contributions}

Lifeng Meng conceived of the research idea. Yonghong Lian and Cuiqiong Li developed the research idea and wrote the manuscript. Jianchun Li and Yongxiang Xie verified the research methods. Qiancheng Liu and Minhua Wu revised the manuscript. Lifeng Meng and Wei Shi encouraged and supervised the findings of this work. All authors reviewed the manuscript.

\section{Funding}


This research is supported by National Natural Science Foundation of China (Grant No.81860838), Guangxi Natural Science Foundation Program (Grant No. 2020JJA140292 and No. 2017JJA140363y).

\section{Availability of data and materials}

All data generated or analyzed during this study are included in this published article.

\section{Ethics approval and consent to participate}

Not applicable

\section{Consent for publication}

Not applicable.

\section{Competing interests}

The authors declare that the research was conducted in the absence of any commercial or financial relationships that could be construed as a potential conflict of interest.

\section{Author details}

Corresponding author: Lifeng Meng: Department of Nephrology, The First Affiliated Hospital of Guangxi University of Chinese medicine, No. 89-9 Dongge Road,Qingxiu District, Nanning City, the Guangxi Zhuang Autonomous Region, China. E-mail address: mlf428@126.com

\section{References}

1. Abbasi M, Chertow G, Hall Y. End-stage Renal Disease. Am Fam Physician. 2010,82 (12):1512.

2. Fresenius Medical Care. ESRD patients in 2012. A global perspective. Germany: Fresenius Medical Care,Deutschland GmbH. 2013. Available from: http://www.vision-fmc.com. Accessed March 15, 2016.

3. Wang WY, Liang Y, Hong, Lu W.Analysis on treatment burden of end-stage renal disease patients and related policy suggestions[J].Chinese Health Resources,2018,21(2):121-126. (in Chinese with English abstract)

4. Liu R, Das B, XiaoW,et al. A Novel inhibitor of homeodomain interacting protein kinase 2 mitigates kidney fibrosis through inhibition of the TGF- $\beta 1 /$ Smad3 pathway. J Am Soc Nephrol, 2017,28 (7):2133-2143.

5. Xiao Z, Chen C, Meng T, et al. Resveratrol attenuates renal injury and fibrosis by inhibiting transforming growth factor-pathway on matrix metalloproteinase 7. Experimental Biology\&M edicine,2016,241(2):140-146.

6. Wang LQ, Zhang WW, Ma MZ, et al. ROS induces epithelial-mesenchymal transition via the TGFB1/PI3K/AKT/mTOR pathway in diabetic nephropathy. Experimental and Therapeutic Medicine. 
2019,17(1):835-846.

7. Solan JL, Lampe PD. Connexin43 phosphorylation: Structural changes and biological effects[J]. Biochem J. 2009;419(2):261-272.

8. Sala G, Badalamenti S.The Renal Connexome and Possible Roles of Connexins in Kidney Diseases[J].American journal of kidney diseases: the official journal of the National Kidney Foundation,2016,67(4): 677-687.

9. Dai P, Nakagami T, Tanaka H, et al. Cx43 mediates TGF-beta signaling through competitive Smads binding to microtubules. Molecular Biology of the Cell, 2007,18:2264-2273.

10. Stains JP, Civitelli R. Gap junctions regulate extracellular signal-regulated kinase signaling to affect gene transcription[J].Mol Biol Cell 2005; 16: 64-72.

11. Ren $\mathrm{S}$, Zhang $\mathrm{H}, \mathrm{Mu}$ YP, et al. Pharmacological effects of Astragaloside IV: a literature review[J]. Journal of Traditional Chinese Medicine,2013,33(3).

12. Wang L, Chi YF, Yuan ZT, et al. Astragaloside IV inhibits renal tubulointerstitial fibrosis by blocking TGF-beta/Smad signaling pathway in vivo and in vitro. Experimental Biology and Medicine(Maywood), 2014, 239(10): 1310-1324.

13. Zhu J. Astragaloside IV inhibits TGF- $\beta 1$-induced epithelial-mesenchymal transition through inhibition of the PI3K/Akt/NF-kB pathway in gastric cancer cells[J].Phytotherapy research: PTR, 2018.DOI:10.1002/ptr.6057.

14. Ryoo IG, Shin DH, Kang KS, et al. Involvement of Nrf2-GSH signaling in TGF $\beta 1$-stimulated epithelialto-mesenchymal transition changes in rat renal tubular cells.2015,38(2):272-281.

15. Vallée A, Lecarpentier Y. TGF- $\beta$ in fibrosis by acting as a conductor for contractile properties of myofibroblasts[J]. Cell and Bioscience,2019,9:98. https://doi.org/10.1186/s13578-019-0362-3

16. Meng XM, Nikolic-Paterson D, Lan H.TGF- $\beta$ : the master regulator of fibrosis[J].Nature Reviews Nephrology, 2016,(12):325-338.

17. Xu J, Lamouille S, Derynck R.TGF- $\beta$-induced epithelial to mesenchymal transition[J]. Cell Research,2009,19:156-172.

18. Kattla JJ, Carew RM, Heljic M, et al. Protein kinase B/Akt activity is involved in renal TGF- $\beta 1$-driven epithelial-mesenchymal transition in vitro and in vivo[J]. American Journal Of Physiology-renal Physiology, 2008, 295:F215-225.

19. Han Q, Lin I,Zhao B, et al. Inhibition of mTOR ameliorates bleomycin-induced pulmonary fibrosis by regulating epithelial-mesenchymal transition[J]. Biochemical And Biophysical Research Communications, 2018,500(4):839-845.

20. Chevallier D, Carette D, Gilleron J, et al. The emerging role of connexin 43 in testis pathogenesis[J]. Curr Mol Med,2013,13(8):1331-1334.

21. Saez JC, Retamal MA, Basilio D, et al. Connexin-based gap junction hemichannels: gating mechanisms[J]. Biochim Biophys Acta, 2005, 1711(2):215-224. 
22. James CC,Zeitz MJ,Calhoun PJ,et al.Altered translation initiation oflimits gap junction formation during epithelial-mesenchymal transition[J]. Molecular biology of the cell,2018,DOI:10.1091/ mbc.E17-06-0406.

23. Chen XM. High glucose-induced hypertrophy of mesangial cells is reversed by connexin43 overexpression via PTEN/Akt/mTOR signaling[J]. Nephrology Dialysis Transplantation, 2012, 27(1):90-100.

24. Kuang JY, Guo YF, Chen Y, et al. Connexin 43 C-terminus directly inhibits the hyperphosphorylation of Akt/ERK through protein-protein interactions in glioblastoma[J]. Cancer Science, 2018, 109(8):26112622.

25. Xu CJ, Wang PF, Liu Y, et al. Regulatory Effect of PI3K/Akt/mTOR Signal on Autophagy in Idiopathic Pulmonary Fibrosis and Intervention Effect of Astragaloside[J].Chinese Journal of Experimental Traditional Medical Formulae„2017,23(18):75-82 (in Chinese with English abstract).

\section{Figures}

a

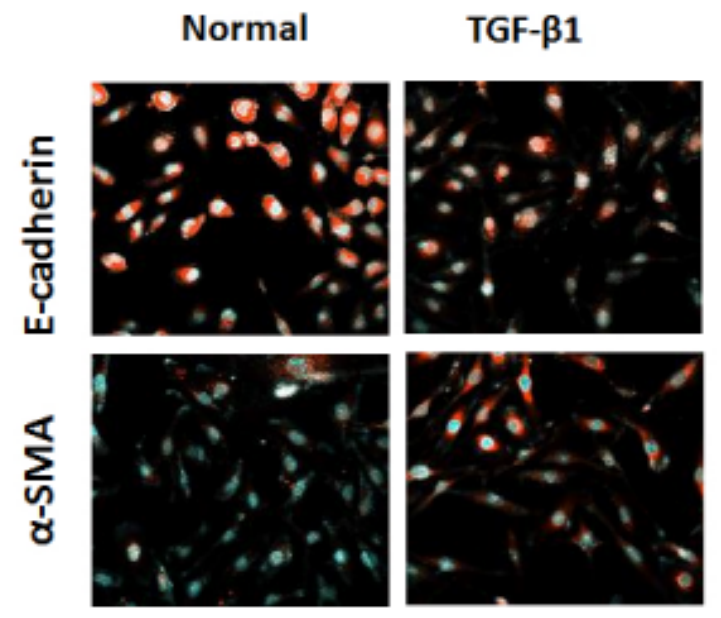

\section{b}

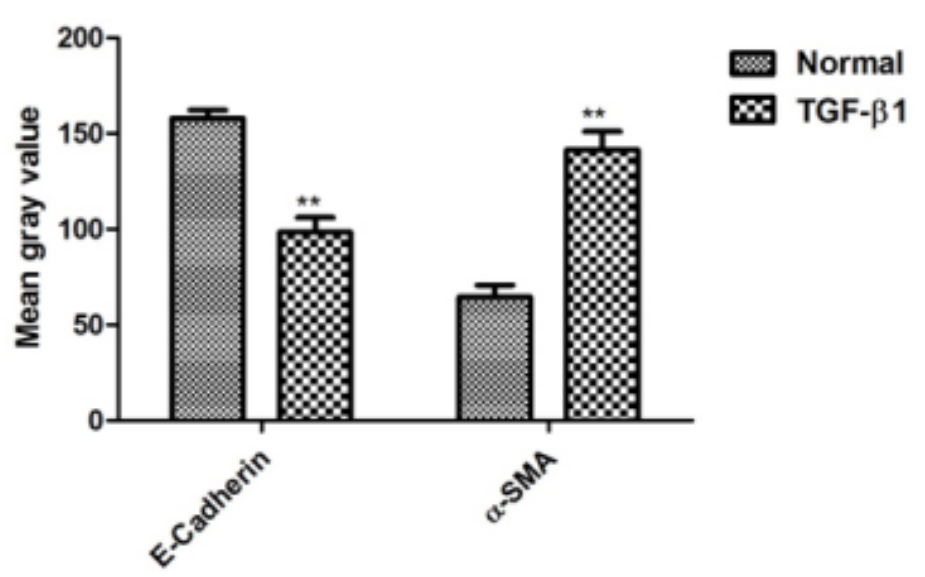

\section{Figure 1}

Comparision of the EMT-related indicators in RTECs before and after being stimulated by TGF- $\beta 1$ RTECs were observed under fluorescence microscope, and the results were shown in Figure 1. After being stimulated by $6 \mathrm{ng} / \mathrm{ml}$ TGF- $\beta 1$ for $48 \mathrm{~h}$, E-cadherin in the model group was fractured and the expression level significantly decreased compared with the normal group $(P<0.01)$ (Figure $1-a, b)$, and the expression level of a-SMA significantly increased $(P<0.01)$ (Figure $1-a, c)$. 
a

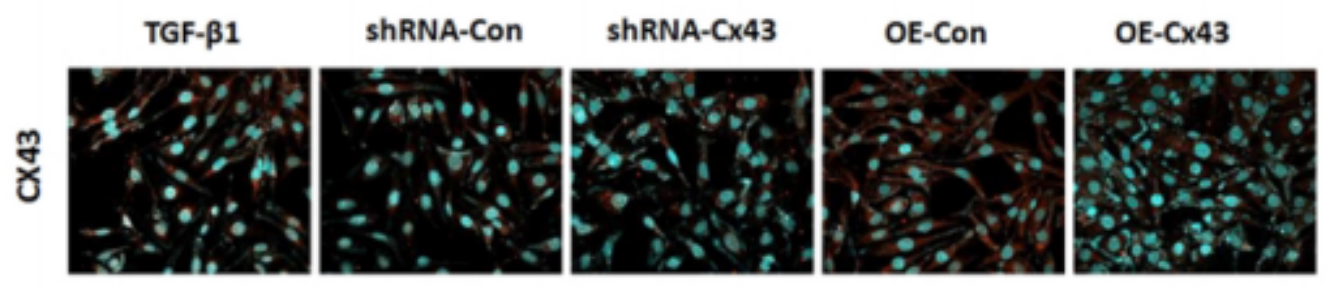

b

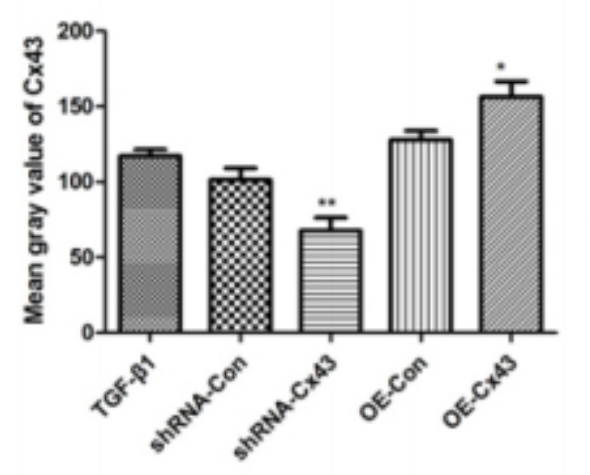

C

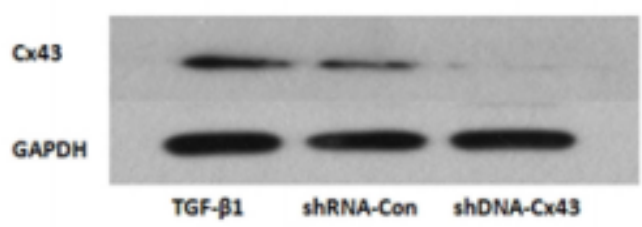

d

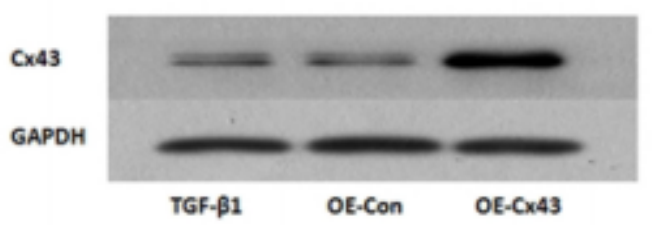

e

$\mathbf{f}$
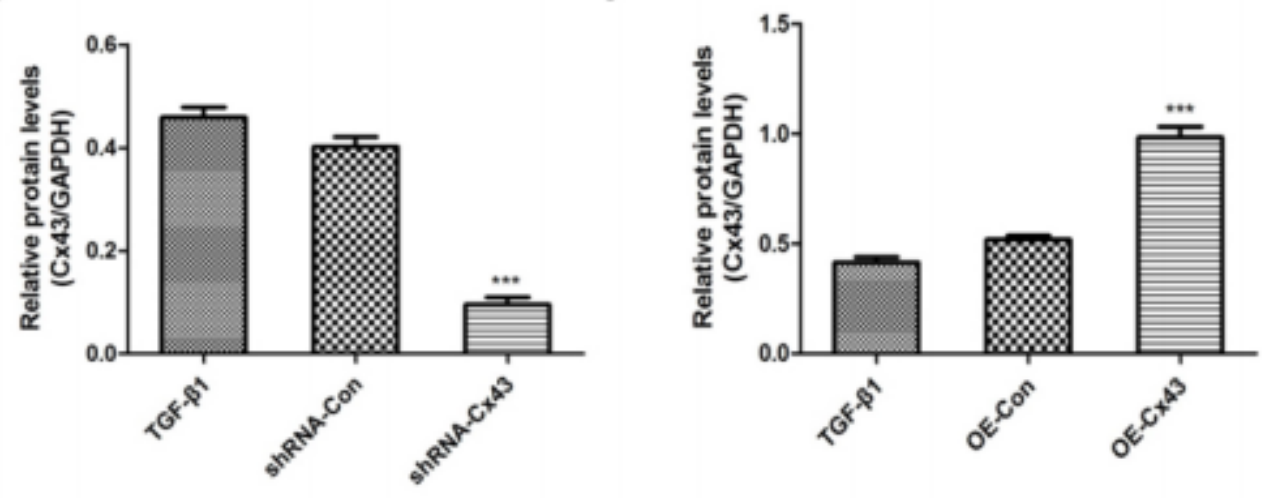

Figure 2

Comparisions of the expressions of Cx43 in RTECs before and after lentiviral transfection Lentiviral vector was transfected into RTECs to interfere with Cx43 specifically and the effect was observed under immunofluorescence microscope. The expression of $\mathrm{C} \times 43$ in shRNA-Cx43 group transfected with lentiviral vector was significantly decreased compared with the model group $(P<0.001)$, while shRNA-Con group, which was the negative control group, showed no significant change in $\mathrm{Cx} 43$ expression compared with the model group $(\mathrm{P}>0.05)$ (Figure 2-a,b). In the OE-Cx43 group transfected with overexpressed lentivirus-interfering vector, the expression of $\mathrm{Cx} 43$ was significantly increased compared with the model group $(P<0.001)$ while in the OE-Cx43 group, the expression of $\mathrm{Cx} 43$ was not significantly changed $(P>0.05)$ (Figure 2-a,b). Western blotting also confirmed the process, which indicating that the lentivirus interference could effectively regulate the expression of $\mathrm{Cx} 43$ (Figure 2-c, d,e,f). 
a

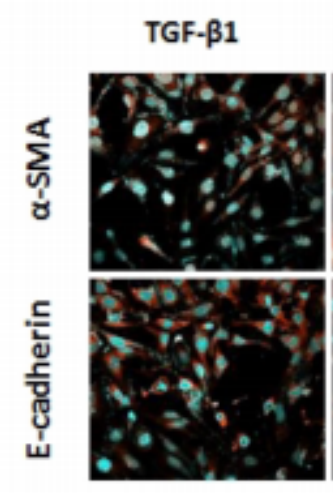

d

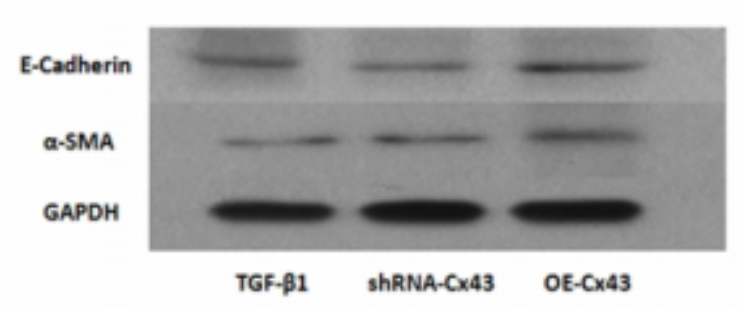

b

C
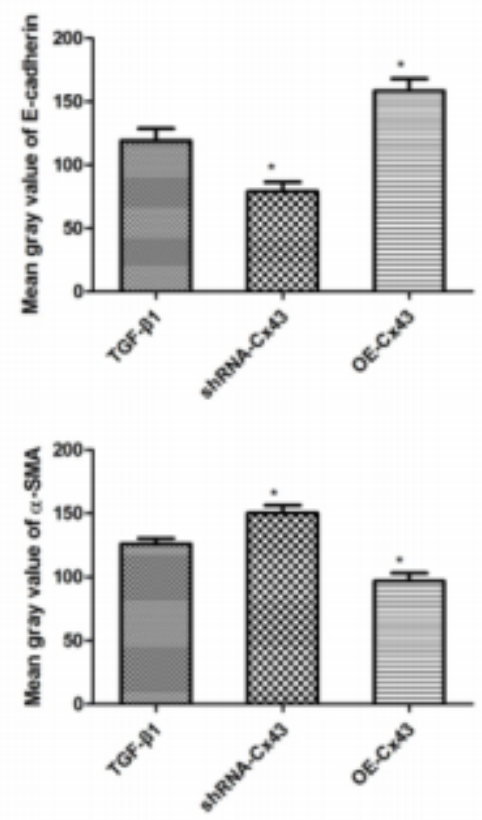

e

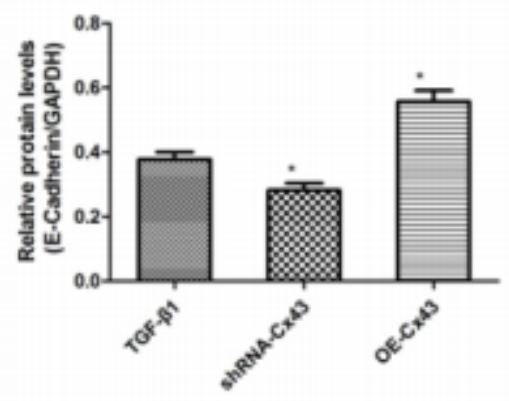

f

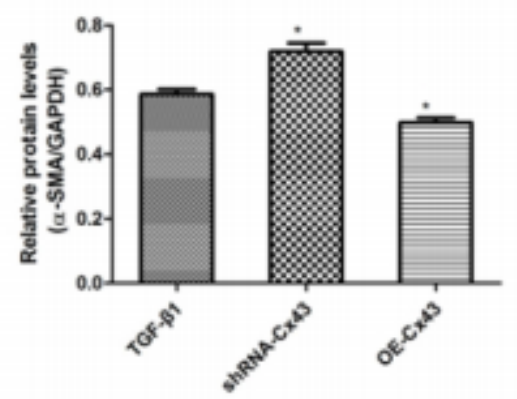

\section{Figure 3}

Comparisions of the EMT-related indicators in RTECs before and after lentiviral transfection The lentivirus shRNA-Cx43 was used to decrease the expression of Cx43. Immunofluorescence results showed that compared with the model group, E-cadherin expression was significantly decreased in shRNA-Cx43 group $(\mathrm{P}<0.05)$ (Figure $3-\mathrm{a}, \mathrm{b})$, while a-SMA expression was significantly increased in shRNA-Cx43 group $(P<0.05)$ (Figure 3-a,c). These were consistent with the changes in the expression of each indicator detected by western blot (Figure 3- d,e,f), indicating that when the expression of $\mathrm{Cx} 43$ was downregulated, the degree of EMT induced by TGF- $\beta 1$ in RTECs was more obvious. On the contrary, lentivirus $\mathrm{OE}-\mathrm{C} \times 43$ was used to reduce the expression of $\mathrm{Cx} 43$. Compared with the model group, the expression of E-cadherin in OE-Cx43 group increased significantly $(\mathrm{P}<0.05)$ (Figure 3-a,b), and the expression of $a-S M A$ decreased significantly $(P<0.05)$ (Figure $3-a, c)$. These were consistent with the changes in the expression of each indicator detected by western blot (Figure 3-d,e,f), indicating that when $\mathrm{Cx} 43$ expression was upregulated, the degree of EMT induced by TGF- $\beta 1$ was inhibited. All these suggested that Cx43 might be an important regulatory molecule involved in the process of EMT induced by TGF- $\beta 1$. 
a

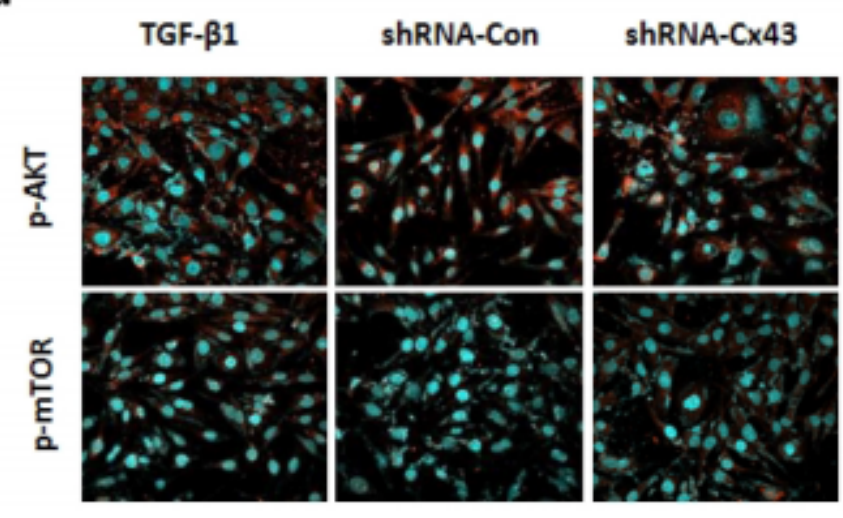

d

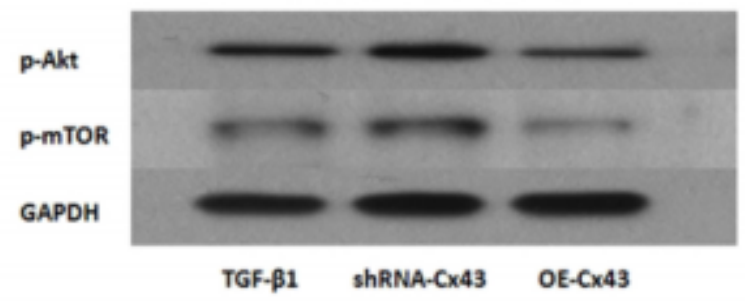

b

C
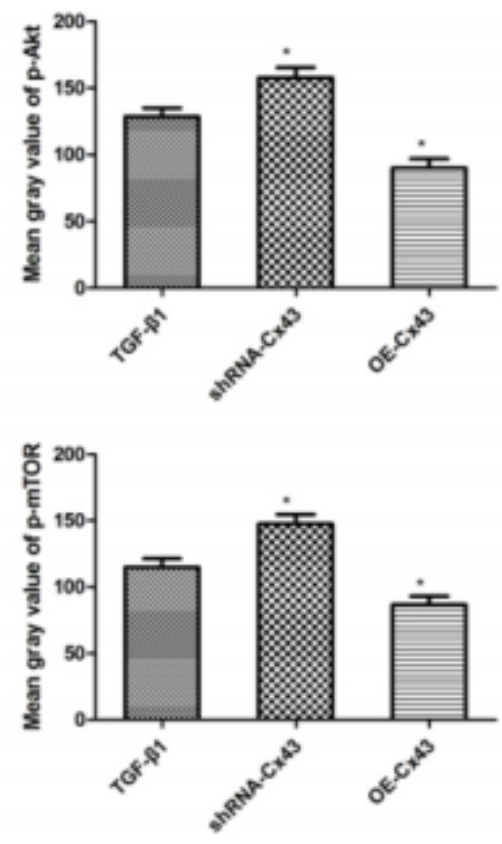

e

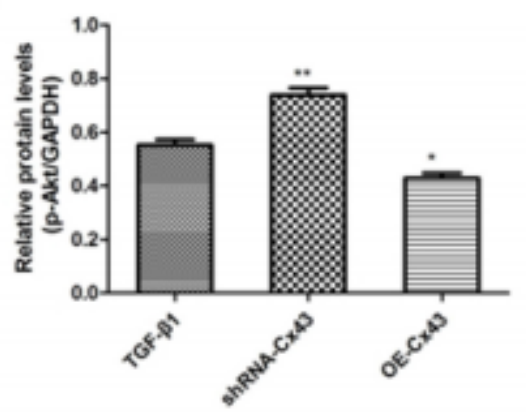

f

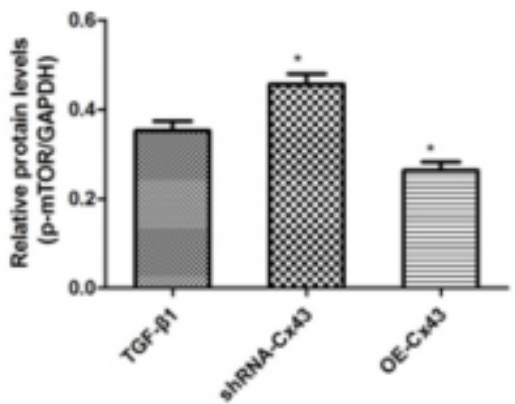

\section{Figure 4}

Comparisions of the phosphorylation levels of Akt and mTOR in RTECs before and after lentiviral transfection Under the immunofluorescence microscope, it was found that when the expression of $\mathrm{Cx} 43$ was reduced by lentiviral vector transfected into RTECs, the phosphorylation levels of Akt and mTOR were significantly increased compared with the model group (Figure 4-a,b,c), and the differences were statistically significant $(P<0.05)$. These were consistent with the changes in the expression of each indicator detected by western blot (Figure 4-d,e,f), which suggested that the EMT induced by TGF- $\beta 1$ in RTECs mignt be related to the activation of Akt/mTOR pathway when the expression of $\mathrm{Cx} 43$ was decreased. However, when the expression of $\mathrm{Cx} 43$ was increased by lentiviral vector transfection, the phosphorylation levels of Akt and mTOR were significantly reduced in the OE-Cx43 group compared with the model group (Figure 4-a,b,c), with statistically significant differences $(P<0.05)$. These were consistent with the changes in the expression of each indicator detected by western blot (Figure 4-d,e,f), which suggested that the EMT induced by TGF- $\beta 1$ in RTECs was alleviated when Cx43 expression was upregulated, which might be related to the inhibition of Akt/mTOR pathway. 


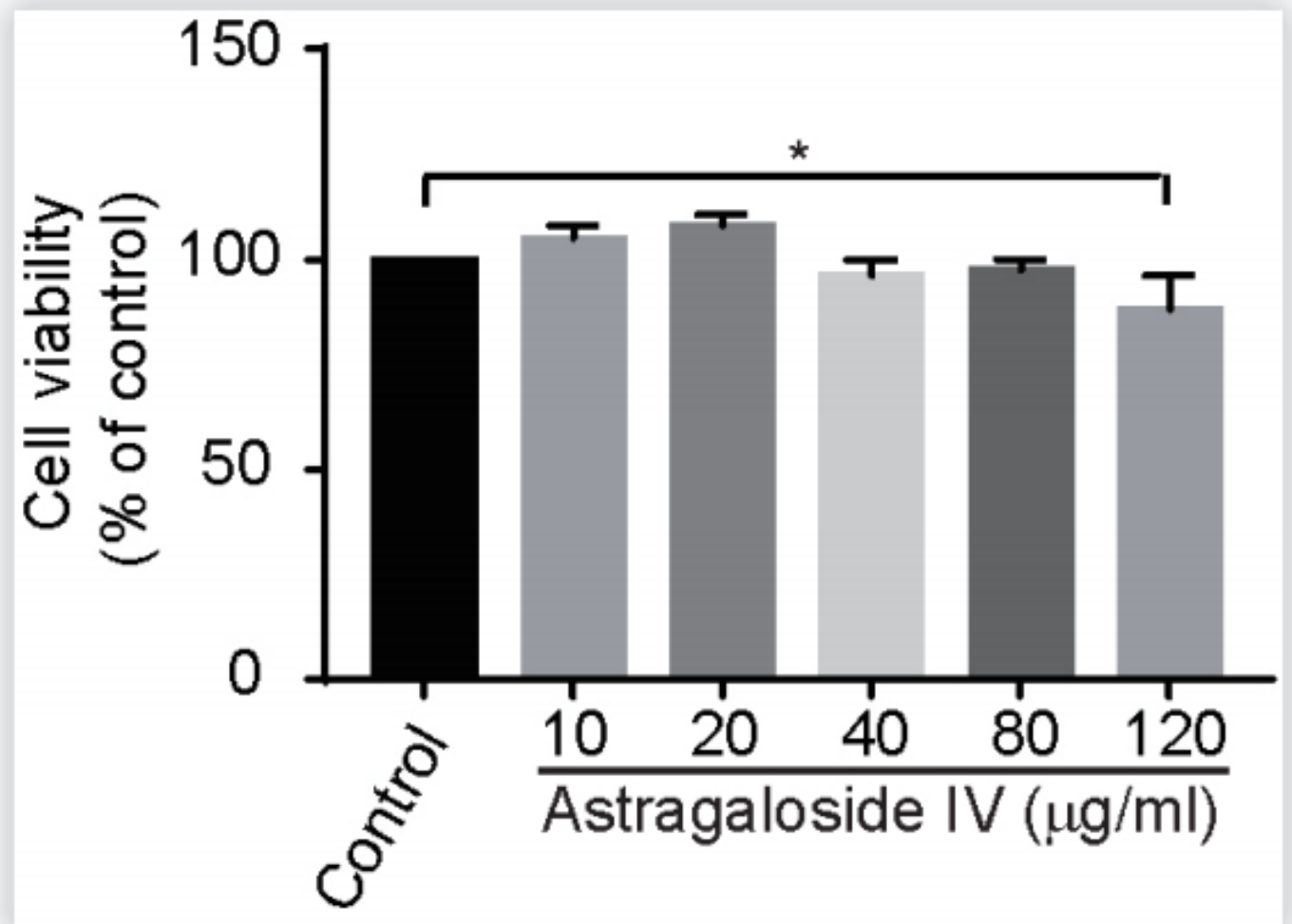

Figure 5

Determination of the effective concentration of ASV CCK-8 assay was employed to examine the effects of ASV with different concentration $(10,20,40,80,120 \mu \mathrm{g} / \mathrm{ml})$ on RTECs' cell viability. The cell viability of the normal control group was defined as $100 \%$, and the ratio to the normal control group was the cell viability of the group stimulated by ASV at different concentrations. When the concentrations of ASV were from 10 to $80 \mu \mathrm{g} / \mathrm{ml}$, the cell activities increased or decreased compared with the normal control group, but the differences were not statistically significant $(P>0.05)$. When the concentration of ASV was $120 \mu \mathrm{g} / \mathrm{ml}$, the cell viability was significantly reduced and the difference was statistically significant $(P<0.05)$ (Figure 5). Therefore, $10 \mu \mathrm{g} / \mathrm{ml}, 40 \mu \mathrm{g} / \mathrm{ml}$ and $80 \mu \mathrm{g} / \mathrm{ml}$ were selected as the low, medium and high intervention concentrations for ASV within the concentration range. 


\section{Image not available with this version}

\section{Figure 6}

Comparisions of the effects of different doses of ASV on RTECs induced by TGF- $\beta 1$ Immunofluorescence assay was used to detect the expression of EMT-related marker proteins E-cadherin and $\alpha$-SMA. Compared with the model group, the expression levels of Cx43 and E-cadherin increased after intervention with $10 \mu \mathrm{g} / \mathrm{ml}$ of ASV (Figure 6-a,b,c), while the expression levels of a-SMA decreased (Figure $6-a, d)$, but the difference was not statistically significant $\triangle P>0.05)$. After intervention with $40 \mu \mathrm{g} / \mathrm{ml}$ and $80 \mu \mathrm{g} / \mathrm{ml}$ of ASV, compared with the model group, the expression levels of $\mathrm{C} \times 43$ and E-cadherin increased (Figure 6-a,b,c) $(P<0.05)$, and the expression levels of a-SMA decreased (Figure 6-a,d) $(P<0.01)$, with statistically significant differences. Consistent with the immunofluorescence results, the effect was also confirmed by western blotting (Figure 6-e,f,h). The protein expressions of $\mathrm{Cx} 43$ was shown in Figure 6-i and Figure 6-I. Compared with the normal control group, the expression level of $\mathrm{Cx} 43$ in the model group was significantly decreased after $6 \mathrm{ng} / \mathrm{ml}$ TGF- $\beta 1$ stimulated for $48 \mathrm{~h}$. While, after intervention with lowdose, medium dose and high-dose of ASV, the expression levels of Cx43 increased compared with the model group, and the effect showed obvious concentration dependence. As shown in Figure 6-i, 6-j and 6$k$, there were no significant differences in the protein expressions of Akt and mTOR in the normal group, model group, low-dose of ASV group, medium-dose of ASV group, and high-dose of ASV group, while the protein expressions of $p$-Akt and p-mTOR were significantly changed. Compared with the normal control group, p-Akt /Akt and p-mTOR /mTOR were increased in the model group, while the ratios of p-Akt/Akt and $\mathrm{p}-\mathrm{mTOR} / \mathrm{mTOR}$ were slightly decreased after intervention with low-dose and medium-dose of ASV, with no statistically significant difference $(P>0.05)$. While after intervention with high-dose of $A S V, p-$ Akt/Akt and p-mTOR /mTOR were significantly reduced, and the difference was statistically significant $(\mathrm{p}<0.01)$. 